\title{
Neuroimaging as a New Diagnostic Modality in Amyotrophic Lateral Sclerosis
}

\author{
Esther Verstraete • Bradley R. Foerster
}

Published online: 20 March 2015

(C) The American Society for Experimental NeuroTherapeutics, Inc. 2015

\begin{abstract}
Amyotrophic lateral sclerosis (ALS) is characterized by progressive degeneration of upper and lower motor neurons, with variable involvement of extramotor brain regions. Currently, there are no established objective markers of upper motor neuron and extramotor involvement in ALS. Here, we review the potential diagnostic value of advanced neuroimaging techniques that are increasingly being used to study the brain in ALS. First, we discuss the role of different imaging modalities in our increasing understanding of ALS pathogenesis, and their potential to contribute to objective upper motor neuron biomarkers for the disease. Second, we discuss the challenges to be overcome and the required phases of diagnostic test development to translate imaging technology to clinical care. We also present examples of multidimensional imaging approaches to achieve high levels of diagnostic accuracy. Last, we address the role of neuroimaging in clinical therapeutic trials. Advanced neuroimaging techniques will continue to develop and offer significant opportunities to facilitate the development of new effective treatments for ALS.
\end{abstract}

Keywords ALS · Neuroimaging · Diagnostic testing $\cdot$ MRI · PET $\cdot$ Biomarker

\section{E. Verstraete}

Department of Neurology, Rudolf Magnus Institute of Neuroscience,

University Medical Centre Utrecht, Utrecht, The Netherlands

B. R. Foerster $(\bowtie)$

Department of Radiology, University of Michigan, 1500 East

Medical Center Drive, Ann Arbor, MI 48109, USA

e-mail: compfun@umich.edu

B. R. Foerster

Ann Arbor VA Healthcare System, Ann Arbor, MI, USA

B. R. Foerster

Russell H. Morgan Department of Radiology, Johns Hopkins

University, Baltimore, MD, USA

\section{Introduction}

Amyotrophic lateral sclerosis (ALS) is a progressive disease of the motor system affecting upper motor neurons in the brain and lower motor neurons in the brainstem and spinal cord, with variable involvement of extramotor brain regions [1]. Each patient differs in their body region of onset, disease progression, and the combination of upper and lower motor neurons involved, meaning that onset and disease course in ALS is heterogeneous $[2,3]$. Besides clinical heterogeneity, underlying pathophysiology in ALS seems to be similarly variable. Monogenetic traits have been identified in familial cases, as well as complex traits with contributions from multiple genetic and environmental factors [4]. Additionally, the same genetic error can result in different clinical phenotypes, while different genotypes can produce the same clinical phenotype. Despite the apparent complexity of ALS, neuroimaging could potentially provide objective phenotypic classification of patients. Such brain phenotyping would provide information on affected brain regions and identify brain alterations associated with different rates of symptom progression. Alongside additional diagnostic information, neuroimaging could serve as a biomarker of disease stage and could monitor progressive degenerative changes in the brain.

Currently, the diagnosis of ALS is based on clinical examination, complemented by objective assessment of the lower motor neurons using electromyography [5]. Involvement of upper motor neurons is assessed by documentation of abnormal reflexes or spasticity during the clinical examination. However, spasticity may not aid diagnosis as it affects only $4 \%$ of patients with ALS at first presentation [6]. Furthermore, lower motor neuron pathology can mask upper motor neuron signs, decreasing their detectability. This is particularly true of patients diagnosed with progressive muscular atrophy (PMA), for whom upper motor neuron degeneration is evident only on postmortem examination [7]. Diagnostic accuracy for 
ALS would improve if upper motor neuron deficits could be established objectively [8]. Current clinical practice uses neuroimaging to exclude conditions with similar clinical presentations to ALS, but not to either confirm or facilitate the diagnosis of ALS. An objective upper motor neuron imaging marker could shorten diagnostic delay, which is still considerable in ALS, and reduce the proportion of misdiagnosed cases [9].

Furthermore, imaging in ALS could identify the involvement of extramotor brain regions, such as degenerative changes in frontotemporal brain regions. Such changes are associated with behavioral and cognitive deficits, which are mild in up to $32 \%$ of cases and moderate to severe in up to $19 \%$ of cases [10]. These cognitive and behavioral deficits are similar to those found in frontotemporal dementia (FTD), which forms a clinicopathological spectrum with ALS as up to $15 \%$ of patients with ALS meet the criteria for FTD [9]. Such deficits are currently assessed using neuropsychological testing of patients and questionnaires for caregivers. However, quantitative imaging measures could increase accuracy of detection, and could identify patients with subclinical extramotor involvement who may be at risk for later development of cognitive and behavioral symptoms.

Imaging has become a vital component of the diagnostic workup for many other neurologic diseases, including multiple sclerosis (MS). Imaging can also guide clinical management of patients once diagnosis has been established. For example, increased white matter lesions imaged with magnetic resonance imaging (MRI) have been shown to precede clinical disease progression in MS, and so provide useful prognostic information [11]. Subsequently, MRI provides an important biomarker in clinical trials for MS, allowing more rapid drug screening. Moreover, MRI has improved the diagnosis, disease monitoring, and efficiency of clinical trials in MS, and is as an example of the successful use of imaging to improve research and clinical care in neurologic diseases.

In this review, we discuss the application of neuroimaging techniques in the study of ALS. We focus on the most promising potential diagnostic markers, describing the steps necessary to develop such biomarkers. We also review some early promising studies, and discuss how such efforts may contribute to improved therapies for patients with ALS.

\section{Measures of Brain Structure}

Brain structure can be examined using high-resolution structural imaging techniques, including MRI, to measure focal loss of gray and/or white matter. Data obtained from these techniques can be analyzed using 2 main approaches: voxelbased morphometry (VBM) and surface-based morphometry $(\mathrm{SBM})[12,13]$. The acquired image data are normalized to reference templates using automated postprocessing software.
Subsequently, the images are segmented into gray and white matter, enabling quantification of brain volume (VBM) and cortical thickness (SBM).

Diffusion tensor imaging (DTI) can provide a measure of the integrity of white matter tracts by detecting the direction and extent of water movement. In healthy, organized tracts, water movement is directional, whereas in diseased, disorganized tracts it is less so [14]. DTI provides several water diffusivity measures by quantifying water movement along 3 perpendicular axes. The mean diffusivity is calculated by averaging the water diffusivity from these 3 axes. In ALS, the most commonly reported DTI measurement is fractional anisotropy (FA). This provides an overall measurement of the directionality, otherwise known as anisotropy, of water diffusion along the 3 axes. This can be further broken down into radial diffusivity, which measures diffusion perpendicular to the axon sheath ( 2 axes), and axial diffusivity, which measures diffusion parallel to the axon sheath (1 axis).

\section{Volumetric Imaging}

VBM studies of patients with ALS have demonstrated volumetric decreases in gray matter. However, the regions implicated vary, with some reporting decreased gray matter in motor and extramotor regions [15-20], others reporting decreased gray matter in extramotor regions only [21,22], and 1 study reporting no change in either region [23]. These discrepancies may simply reflect differences in the VBM analyses used [24].

To address this variability, the results from 5 VBM studies, comprising 84 patients with ALS and 81 healthy controls, were pooled in a meta-analysis, which reported significant gray matter loss in the right motor cortex of patients [25]. VBM alterations may show specificity for particular clinical presentations. A recent study compared patients with classic ALS, patients with ALS-FTD, and patients with other neurologic diseases, demonstrating reductions in gray matter volume in motor and extramotor regions only in patients with ALS-FTD [26]. A recent longitudinal VBM study reported decreased gray matter volume over 6 months in the motor and frontotemporal regions, thalami, and caudate [27].

Compared with VBM studies, SBM studies of patients with ALS uniformly report reduced cortical thickness in primary motor regions [28-31]. While SBM provides measures of cortical surface area and cortical volume, it has been demonstrated that cortical thickness is the key metric to detect changes in ALS [30]. Cortical thinning has been shown to be a consistent alteration that is specific to upper motor neuron degeneration in ALS. Cortical changes are not seen in disorders that mimic ALS or in patients with PMA (Fig. 1) [32]. In addition, clinical disability in particular body regions correlated with focal cortical changes in corresponding regions of the somatotopic representation in the motor cortex [32], 


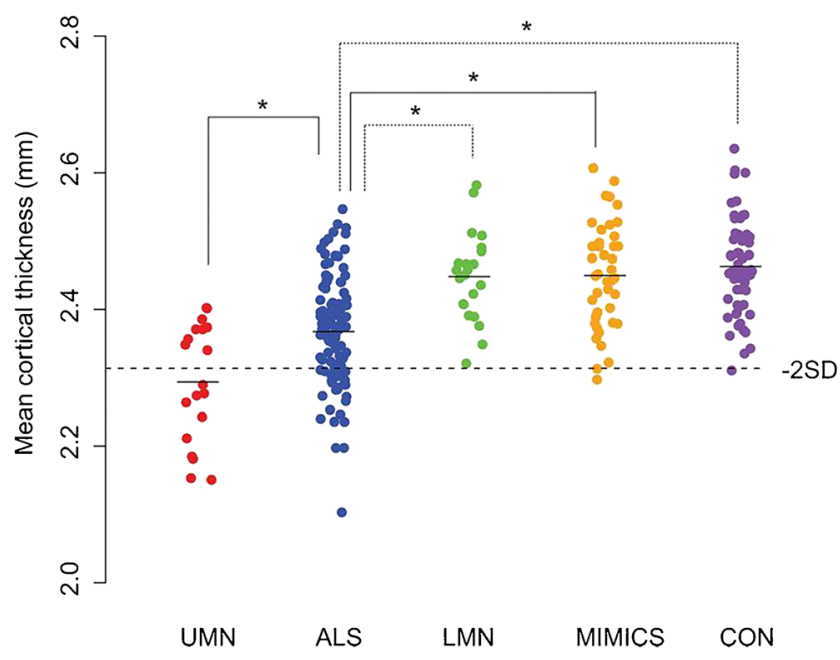

Fig. 1 Cortical thickness of the precentral gyrus in patients with motor neuron disease subtypes compared with mimic disorders and healthy controls. Mean cortical thickness of the precentral gyrus (left and right averaged) was plotted per study group. Cortical thickness of patients with amyotrophic lateral sclerosis (ALS) was significantly reduced compared with patients with a lower motor neuron (LMN) phenotype, mimic disorders, and healthy controls. Patients with a clinical upper motor neuron (UMN) phenotype had a significantly lower cortical thickness compared with patients with ALS. The dashed horizontal line indicates the mean cortical thickness minus 2 SDs of the control group (i.e., controls and mimics combined). MIMIC $=$ mimic disorder; $\mathrm{CON}=$ healthy control. Reproduced with permission from Walhout R, Westeneng HJ, Verstraete E, et al. Cortical thickness in ALS: towards a marker for upper motor neuron involvement. J Neurol Neurosurg Psychiatry 2014;36;1075-1082. Copyright (C) 2014 BMJ Publishing Group Ltd

consistent with a previous VBM study [20]. Newer highresolution analytic methods, such as fractal dimension analysis, are able to model alterations in the shape of white matter, providing an additional potential diagnostic biomarker [33].

In conclusion, the findings outlined here support volumetric imaging, particularly evaluation of cortical thickness, as a promising neuroimaging metric for the development of a diagnostic biomarker.

\section{DTI}

Axonal degeneration and disorganization of white matter in ALS are thought to result in changes in water diffusion parameters [34, 35]. Many studies in ALS have demonstrated water diffusion alterations, the most common being a decrease in FA of the corticospinal tract, the posterior limb of the internal capsule, and the corpus callosum [36]. Patterns of decreased FA along the corticospinal tract differ in patients with ALS with early-stage limb onset compared with patients with early-stage bulbar onset [37]. FA values may also differentiate between ALS and similar disorders, and therefore DTI could aid classification of motor neuron diseases. Patients with primary lateral sclerosis showed the lowest corticospinal tract FA values, while patients with ALS showed intermediate FA reductions; the least extensive FA reductions were seen in patients with PMA. Axial diffusivity and radial diffusivity measures are not as consistent. However, increased axial diffusivity and increased radial diffusivity in the corticospinal tract and increased radial diffusivity in the corpus callosum of patients with ALS have been reported in several studies [38-41]. Furthermore, differences in axial diffusivity of the corticospinal tract have been reported when comparing patients with classic ALS with those with ALS-FTD [42].

Imaging alterations in extramotor regions are reported in the literature, although less commonly than those in the corticospinal tract or corpus callosum [43-47]. Such findings may indicate the involvement of extramotor regions in clinical symptoms, including behavioral and cognitive disturbances. It is possible to classify patients according to stage of disease using patterns of white matter tract alterations that include extramotor regions, with the greatest effect size for FA changes in the corticospinal tract [48].

In summary, the most promising candidates for diagnostic DTI biomarkers are FA changes in the corticospinal tract and corpus callosum. Furthermore, DTI may allow elucidation of the brain phenotype of ALS by detecting white matter tract changes in extramotor regions.

\section{Measures of Brain Receptors and Metabolites}

Positron emission tomography (PET) and magnetic resonance spectroscopy (MRS) can be used to examine alterations in brain receptors and brain metabolites, respectively.

In the ALS literature, there are fewer PET studies than MRI studies, likely owing to the added complexity and costs of PET. Nonetheless, PET offers the unique opportunity to study neuronal receptors and protein expression in ALS [49, 50]. PET can be used to map receptors, including the $\gamma$ aminobutyric $\operatorname{acid}_{\mathrm{A}}\left(\mathrm{GABA}_{\mathrm{A}}\right)$ receptor using $\left[{ }^{11} \mathrm{C}\right]$ flumazenil, and the serotonergic 5-hydroxytryptamine ${ }_{1 \mathrm{~A}}$ receptor using $\left[{ }^{11} \mathrm{C}\right]$ WAY 100635. Another PET agent, $\left[{ }^{11} \mathrm{C}\right] \mathrm{PK} 11195$, binds to translocator proteins and is considered a marker of neuroinflammation.

Proton MRS can provide estimates of neuronal density and function ( $\mathrm{N}$-acetylaspartate), cellular membrane turnover (choline), and energy metabolism (creatine) [51]. Short echo times allow detection of metabolites with short echo relaxation times, including "Glx" (glutamine + glutamate) and myo-inositol, a marker of glial cells. Although whole-brain approaches for MRS are possible, in most cases a prescribed volume, or "voxel", is placed in the brain region of interest. Recently developed MR spectral editing techniques allow for measurement of GABA at $3 \mathrm{~T}$ field strength [52]. 


\section{PET}

Both GABAergic and serotonergic systems have been studied using PET in patients with ALS. Reductions in $\left[{ }^{11} \mathrm{C}\right]$ flumazenil binding in the motor cortex and motor association areas have been demonstrated in patients with sporadic ALS. In contrast, patients with ALS and the "D90A" mutation in SOD1 exhibit reductions in $\left[{ }^{11} \mathrm{C}\right]$ flumazenil binding in the left frontotemporal region and anterior cingulate gyrus [53]. Global cortical binding of $\left[{ }^{11} \mathrm{C}\right]$ WAY 100635 was similarly decreased in patients with ALS with the SOD1 mutation and patients with sporadic ALS (who showed more marked decreases), with the largest change for both groups in frontotemporal regions [54]. There may be different neuronal vulnerabilities for patients with ALS carrying a SOD1 mutation compared with sporadic ALS, leading to differences in their cortical imaging signature. Another PET study reported increased binding of $\left[{ }^{11} \mathrm{C}\right] \mathrm{PK} 11195$ in the motor cortex, dorsolateral prefrontal gyrus, thalamus, and pons of patients with ALS, implicating increased microglial activation indicative of neuroinflammation [55]. However, there was a marked overlap of $\left[{ }^{11} \mathrm{C}\right]$ PK11195 binding for patients with ALS and healthy controls, tempering enthusiasm for use of this PET agent as a diagnostic biomarker.

\section{MRS}

The most commonly reported finding in ALS studies using MRS is reduced $N$-acetylaspartate in the motor cortex, indicating a loss of neuronal integrity in this region [56]. Additionally, MRS studies report increased myo-inositol in the motor cortex of patients with ALS [57-59]. However, reports of Glx alterations in the motor cortex are not consistent, perhaps partly owing to the effects of riluzole, a glutamate antagonist used for ALS treatment. Several studies did not explicitly report whether imaged patients were treated with this medication [57, 60-62]. Reduced GABA levels have been reported in the motor cortex of patients with ALS, which is consistent with the results of an earlier PET $\left[{ }^{11} \mathrm{C}\right]$ flumazenil study $[62,63]$. These results indicate an "interneuronopathy" in which a relative GABA deficiency may contribute to increased glutamatergic activity and subsequent neuronal damage [64]. However, the most promising potential MRS imaging biomarker in ALS is $N$-acetylaspartate in the motor cortex. Glx, myo-inositol, and GABA may also be candidates for diagnostic biomarkers but require additional research to demonstrate the extent of alterations in early disease stages.

\section{Measures of Brain Activity}

PET can also measure blood flow and brain function. The most readily available PET agent is $\left[{ }^{18} \mathrm{~F}\right]$ fluorodeoxyglucose
(FDG), which measures cellular metabolism using a glucose analog. Compared with other PET agents, $\left[{ }^{18} \mathrm{~F}\right] \mathrm{FDG}$ is relatively available for clinical use and does not require an onsite cyclotron for manufacture.

In contrast to PET, which requires a radioligand, functional MRI uses the blood oxygen level-dependent (BOLD) signal from the inherent paramagnetic properties of blood to study brain activity [65]. Hemoglobin in its deoxygenated state is susceptible to the magnetic field, whereas oxygenated hemoglobin is not. Neural activity is accompanied by changes in blood flow and oxygenation, which can therefore be measured noninvasively using MRI as a proxy for brain activation. Functional imaging experiments can measure neural activity related to motor, cognitive, or emotional stimuli.

\section{PET}

An early PET study demonstrated reduced blood flow to the motor cortex in ALS, with activation of the contralateral motor cortex during a motor task, suggesting compensatory recruitment within the motor cortical system [66]. Two $\left[{ }^{18} \mathrm{~F}\right]$ FDG studies of patients with ALS reported increased metabolic activity in extramotor regions, including the upper brain stem, mesial temporal region, and cerebellum [67, 68]. Additionally, decreased metabolism was seen in the frontal brain regions. However, only the larger study reported decreased metabolism in the motor cortex [68]. A more recent study reported reduced metabolism in the frontal, motor, and occipital regions, and increased metabolism in the midbrain, temporal pole, and hippocampus in patients with ALS [69]. In 2 studies, patients with ALS could be differentiated from healthy controls with a high degree of diagnostic accuracy using $\left[{ }^{18}\right.$ F]FDG $[68,69]$. Undoubtedly, continued development of new PET agents will provide more potential biomarkers for ALS.

\section{Functional MRI}

Functional MRI (fMRI) studies of motor function complement earlier PET results by demonstrating increased recruitment of cortical regions associated with motor processing, suggesting that the ALS brain attempts to compensate for functional loss [70, 71]. Recently, abnormalities of prefrontal activation to letter fluency have been reported in both patients with PMA and ALS [72]. fMRI using tasks to investigate cognitive and behavioral performance can provide insights into processes, such as functional reorganization. However, for reasons of quantification and standardization it is unlikely that this type of measure will develop diagnostic markers. The limitations of task-driven fMRI are particularly evident in the context of ALS as the patient's level of disability directly impairs his or her ability to perform the given task, resulting 
in significant challenges to interpreting the resulting imaging data.

\section{Network-Based Analyses}

The brain is a complex neural network, consisting of structurally and functionally interconnected regions at many levels [73]. Each brain region has a certain degree of functional specialization but does not function independently. As described above, structural and functional studies have shown regional gray and white matter changes in ALS. Most changes involve motor regions, but altered extramotor regions are also reported. Network-based analyses explore the changes in structural and functional connectivity within brain networks at a system-wide level, to better understand the relationship between motor and extramotor alterations seen in the ALS brain.

\section{Functional Connectivity Resting-State fMRI}

Resting-state fMRI (rs-fMRI) provides a measure of basal brain activity using BOLD imaging techniques. Using the rs-fMRI data, functional connectivity of the brain is then evaluated based on the degree of synchronization of lowfrequency oscillations between anatomically separated brain regions $[73,74]$. Research has shown that patterns of correlated activity in the human brain reflect functional communication between neuronal populations of anatomically separated brain regions $[75,76]$. Functional connectivity studies of ALS have used different methodological approaches, some focusing only on the sensorimotor network or structurally affected regions (e.g., defined by VBM), while others use a wholebrain approach.

The first rs-fMRI study evaluated 5 resting state networks, reporting reduced connectivity in the default mode and sensorimotor networks [77]. The default mode network comprises widespread areas throughout the brain, including the medial frontal regions, parietal regions, inferior temporal gyrus, cingulate cortex, and precuneus, and is consistently deactivated during the performance of cognitive tasks [78, 79]. The sensorimotor network is composed of the primary motor cortex, anterior cingulate cortex, somatosensory regions, and auditory cortex [80]. Others report reduced functional connectivity in the motor network $[81,82]$. However, several subsequent studies report increased functional activity between the sensorimotor cortex and extramotor regions [83, 84], or subregions of either increased or decreased functional coherence within the sensorimotor network [85]. A recent study investigated links between functional and structural connectivity in ALS, reporting that these effects change in the same direction. Thus, connections with reduced structural integrity also show reduced functional connectivity [86].
A study found connectivity changes in the default mode and frontoparietal networks in patients with ALS compared with healthy controls, which were associated with measures of cognition and behavior. Enhanced parietal connectivity was associated with cognitive deficits in the patients with ALS, perhaps representing a compensatory mechanism [87]. In line with this study, a recent rs-fMRI study examined both patients with FTD and ALS, showing increased activation in the posterior cingulate cortex in patients with behavioral-variant FTD, while activation in these areas was suppressed in patients with ALS. However, overall functional connectivity patterns in ALS and FTD were strikingly similar [88]. It is expected that techniques with much higher temporal resolutions than fMRI, such as magnetoencephalography, will provide future insights into functional connectivity changes in the context of ALS [89].

\section{Structural Connectivity DTI}

In addition to studying selected white matter tracts, DTI also allows reconstruction of the entire structural brain network. Using network-based statistics, research has shown that patients with ALS have an impaired subnetwork compared with healthy controls. This subnetwork includes bilateral primary motor regions, bilateral supplementary motor regions, regions of the basal ganglia, the left hippocampus, right posterior cingulate, and right precuneus [90]. A recent study applied a similar analysis, using network-based statistics in a different ALS cohort and revealed a subnetwork involving very similar connections [91]. Longitudinal connectivity changes appear to expand with the subnetwork of affected structural connections involving frontal, temporal, and parietal regions (Fig. 2) [92].

Assessing affected subnetworks in individual patients may provide important diagnostic and prognostic information. This would provide information about which connections are involved and the extent to which brain networks are involved. Disease progression could be monitored by assessing changes over time. However, the complexity of this type of analysis may complicate translation to clinical practice.

\section{What Barriers Must Be Overcome?}

Over the last decade, the number of published studies of neuroimaging in ALS has increased considerably. Despite this increase, there are still no clinically meaningful imaging biomarkers for the involvement of upper motor neurons. There are several barriers to overcome to achieve this goal.

First, imaging studies need to progress from group-level analyses to the use of imaging data for prediction at the individual level. Second, to ensure generalizability to the clinic, patients included in imaging studies should closely resemble 
a $\mathrm{T}=1$ $\mathrm{T}=2$
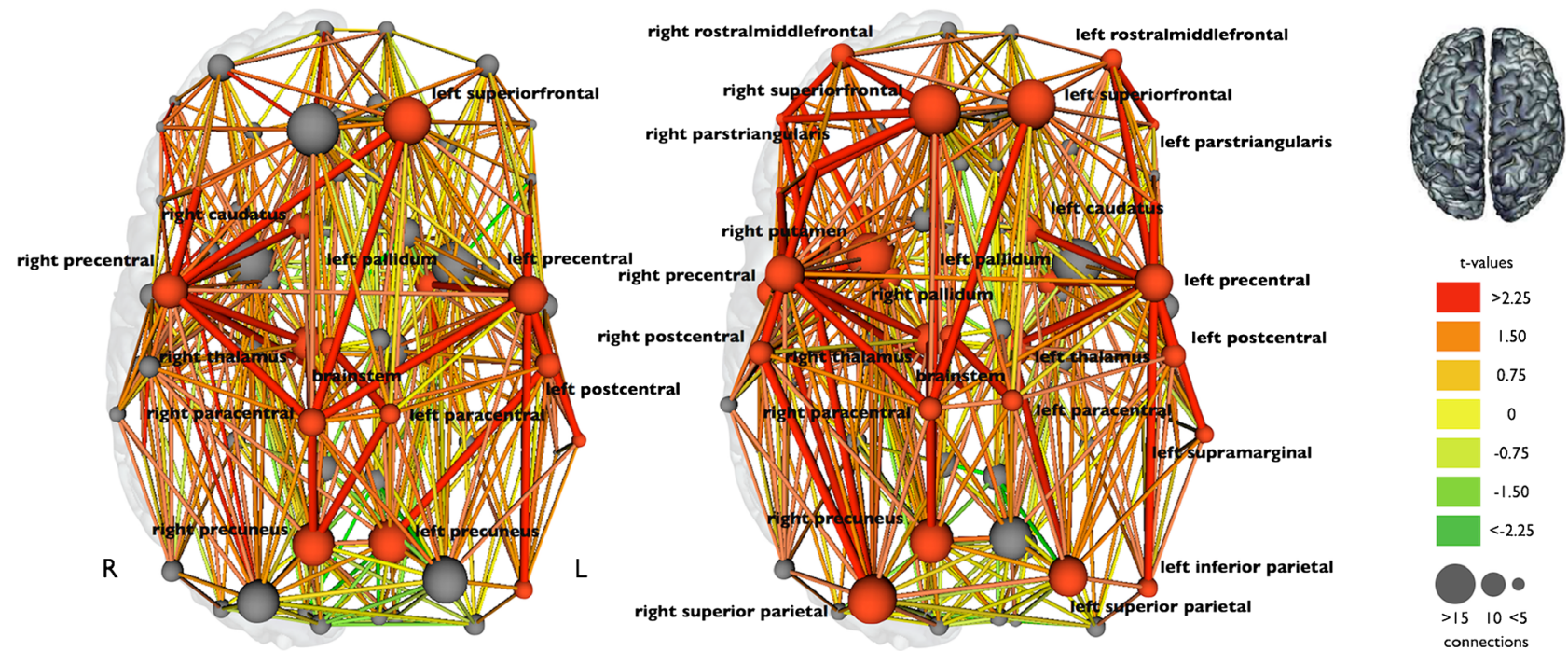

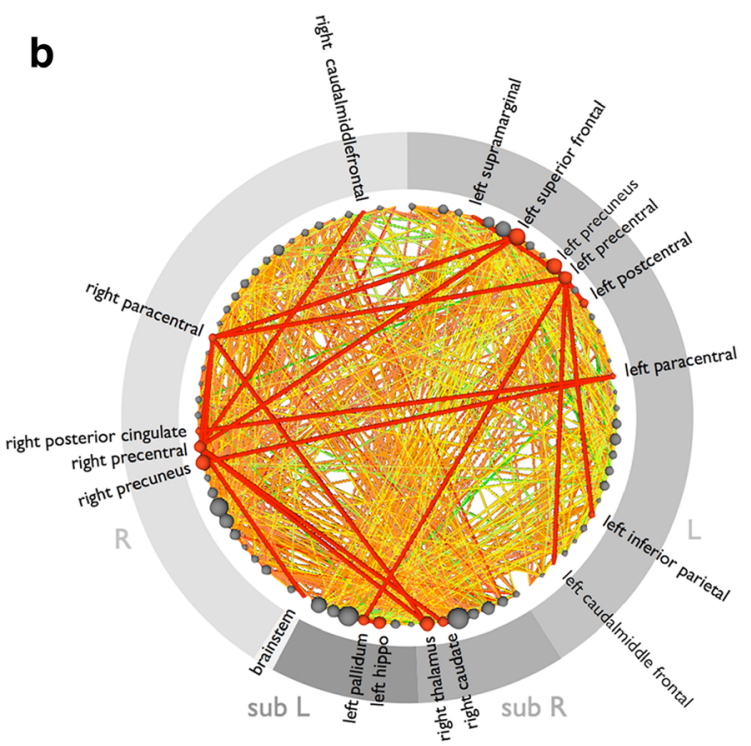

Fig. 2 Longitudinal structural connectivity changes in amyotrophic lateral sclerosis (ALS). This figure shows the reconstructed structural brain networks, and affected connections in 25 patients with ALS compared with a group of healthy controls at 2 time points. a The affected connections and interconnecting nodes are displayed in red comparing patients at baseline and follow-up (after an average of 5.5 months) with a group of healthy controls. The size of the nodes is determined by their degree (number of structural connections). Nodes and connections are red if they are part of the affected subnetwork. The affected subnetwork at follow-up included more structural connections,

patients that are seen routinely in clinical practice. As such, MRI studies of diagnostic markers for ALS should include patient cohorts similar to the patients referred to the neuromuscular clinic, including those with uncertain or alternative diagnoses. Ideally, patients would be imaged as part of the initial diagnostic work-up. To date, the large majority of imaging studies in ALS have studied patients with a relatively

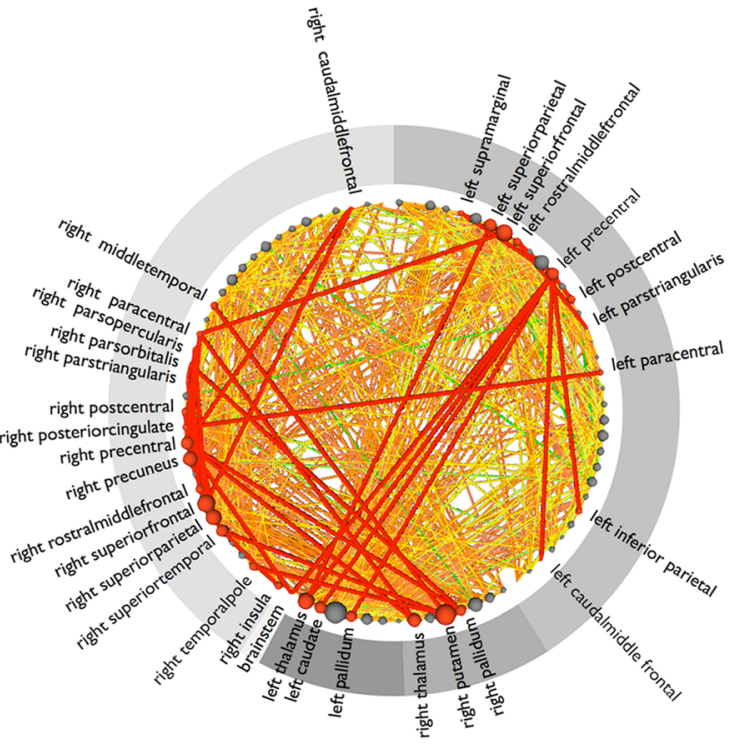

extending mainly to frontal and parietal brain regions. b This figure shows all brain regions arranged on a ring. The red nodes and connections are part of the affected subnetwork, showing an increasing number of affected connections and regions at follow-up compared with baseline. $\mathrm{R}=$ right; $\mathrm{L}=$ left; $\mathrm{sub}=$ subcortical structures; $\mathrm{T}=1=$ first time point; $\mathrm{T}=2=$ second time point. Reproduced with permission from Verstraete E, Veldink JH, van den Berg LH, van den Heuvel MP. Structural brain network imaging shows expanding disconnection of the motor system in amyotrophic lateral sclerosis. Hum Brain Mapp 2014;35:1351-1361. Copyright (C) 2013 Wiley Periodicals, Inc

long disease duration-much longer than the average diagnostic delay of 10-12 months [9]. These patients likely have more advanced disease, which may lead to overestimation of effect sizes. Conversely, patients who remain eligible for inclusion in an MRI study 2 years after an ALS diagnosis may represent a subgroup with relatively slow disease progression, which would be reflected in the imaging results. Additionally, 
it is crucial to include patients with other neurological diagnoses, preferably ALS mimics, in order to establish specificity of the diagnostic biomarker under investigation [93].

\section{Phases of Diagnostic Test Development}

Diagnostic tests must proceed through a series of development phases before they are considered validated for clinical use. Given the time requirements and costs of assessments of new technology, it is pragmatic to evaluate diagnostic tests using a step-wise approach. As in drug development, the proposed diagnostic test in question must meet objective endpoints, as each successive phase requires greater resources. Steps in the development of a proposed diagnostic test can be divided into 4 phases: 1) establish a range of normal values as compared with the diseased state; 2) test diagnostic accuracy under ideal conditions; 3) test diagnostic accuracy under clinically relevant conditions; and 4) determine impact of diagnostic test on clinical outcomes [94, 95].

\section{Phase 1 Studies}

These studies are designed to assess group differences between healthy controls and patients known to have the target disease. If patients with the target disease have a significantly different set of values compared with the range for healthy controls, this provides evidence that the test may hold promise as a diagnostic test, providing justification to proceed to the more resource intensive later phases. These types of studies can provide important understanding about the underlying pathophysiology of the studied disease, and provide new targets for treatment. A common statistical test used for this phase is a $t$ test. The vast majority of ALS neuroimaging studies fall into the phase 1 category.

\section{Phase 2 Studies}

Whereas phase 1 studies focus on group differences, phase 2 studies are targeted at the individual level. Although the same data can be used as in phase 1, phase 2 investigates whether patients with confirmed disease are more likely to have a positive result than healthy controls. Statistical analyses for this phase generally include determination of a receiver operating characteristic (ROC) curve, sensitivity, specificity, and likelihood ratios for the assessment of diagnostic accuracy. Although favorable results at this phase are encouraging, it must be recognized (and reported) that this diagnostic evaluation is done under ideal conditions. In clinical practice, diagnostic tests are ordered for patients for whom a diagnosis has not yet been established. This brings us to phase 3 of diagnostic test development.

\section{Phase 3 Studies}

Phase 3 addresses the clinically relevant question of how well the test in question correctly classifies disease in those patients with suspected disease. For example, a patient might present with lower motor neuron symptoms but no clear upper motor neuron signs. Test results from a neuroimaging study (e.g., FA along the corticospinal tract) could be used to classify patients with or without disease, using the imaging parameter. Longitudinal monitoring of the patient could then assess whether there is a conversion to a diagnosis of ALS. Once again, statistical analyses require determination of a ROC curve, sensitivity, specificity, and likelihood ratios. Phase 3 studies require additional resources and effort compared with phase 2 studies given the challenges inherent in recruiting the appropriate subjects, designing, and executing such study protocols to ensure a clinically relevant spectrum of suspected disease.

\section{Phase 4 Studies}

Ultimately, the key clinical question about a diagnostic test under evaluation is whether it improves outcomes for the patient. Randomization is required to assess classification measures accurately, adding significant cost and complexity to phase 4 studies. In ALS, as in other neurodegenerative diseases, there are no current lifesaving treatments, making the benefit of a diagnostic test less evident. However, new treatments are on the horizon. An earlier diagnosis for patients who fall into the suspected disease category may particularly benefit from new potential treatments. In addition, an accurate diagnostic test has important implications for clinical management and practice. First, it prevents the clinician from performing additional diagnostic tests or unnecessary surgical interventions, which may potentially harm the patient. Second, it shifts the focus towards optimizing disease management. From the patient perspective, a definitive diagnostic test provides added "value in knowing" [96], allowing the patient to seek closure psychologically after a "certain" diagnosis and make life plans. In the case of a potential familial condition, it is important to establish a correct diagnosis more rapidly so the family member can receive genetic counseling.

\section{Analyzing Imaging Data}

\section{Challenges for Analyses}

The attributes of an ideal diagnostic neuroimaging biomarker for ALS would include quick and easy acquisition (i.e., short MRI acquisition time), easy interpretation (i.e., visual depiction of the imaging data with clear findings), and high diagnostic accuracy (i.e., high sensitivity and specificity). An 
example of a neuroimaging biomarker with these attributes is diffusion-weighted imaging for stroke.

Several of the neuroimaging techniques described in previous sections are relatively easy to acquire. However, significant challenges remain for analyzing the imaging data and establishing sufficient diagnostic accuracy. Several phase 2 studies have evaluated the diagnostic accuracy using single neuroimaging modalities, such as DTI and cortical thickness, with encouraging results, although clinical utility is currently limited [28, 30, 32, 58, 97-101]. Using different statistical approaches, two meta-analyses evaluated several DTI studies and found that a single imaging metric results in marginal diagnostic accuracy measures (Fig. 3) [102, 103]. Given high clinical heterogeneity, the involvement of both motor and extramotor systems, and the likely convergence of multiple pathogenic mechanisms in ALS [104], it is becoming increasingly clear that a multidimensional approach for diagnostic neuroimaging biomarkers is necessary.

A multidimensional approach requires advanced statistical methods to identify salient features and develop a "fingerprint" of the disease. Just as in genetics, one of the key challenges in performing imaging analysis is that the datasets consist of large amounts of data derived from relatively small sample sizes. For example, a resting-state fMRI dataset from a single patient can consist of thousands of potentially important variables that must be analyzed. Traditionally, such large

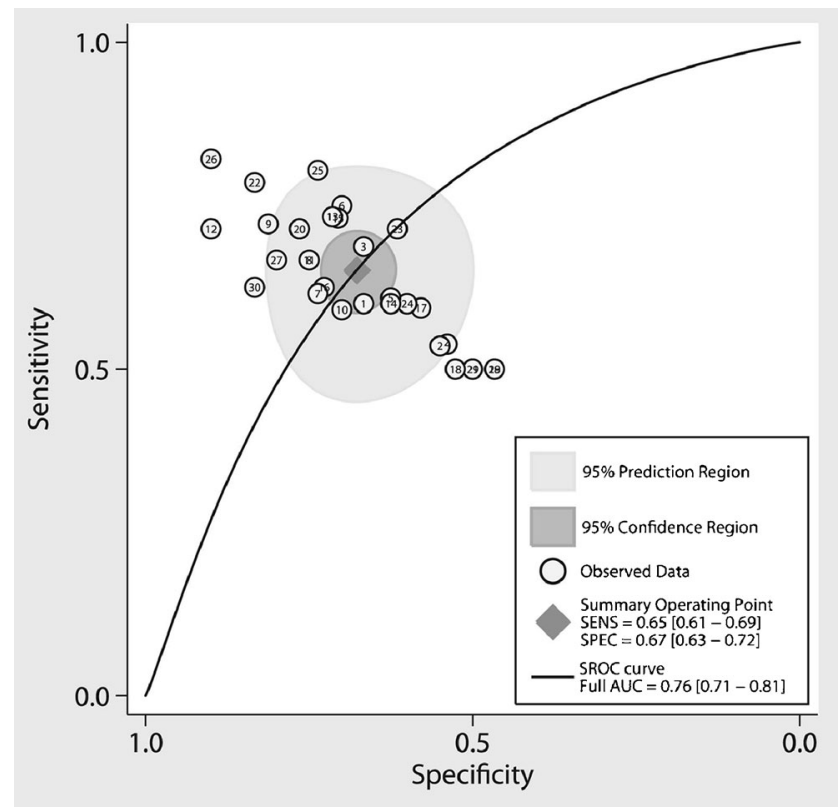

Fig. 3 Meta-analysis evaluating diagnostic accuracy of diffusion tensor imaging (DTI) for ALS. The summary operating point (diamond) represents the intersection of overall sensitivity and specificity. AUC = area under the curve; $\mathrm{SROC}=$ summary receiver operator curve; $\mathrm{SENS}=$ sensitivity; SPEC = specificity. Reproduced with permission from Foerster BR, Dwamena BA, Petrou M, et al. Diagnostic accuracy using diffusion tensor imaging in the diagnosis of ALS: a meta-analysis. Acad Radiol 2012;19:1075-1086. Copyright (C) 2012 Elsevier, Inc datasets have been analyzed using univariate approaches, such as statistical parametric mapping. Here, a single variable (such as voxel intensity, derived from the BOLD signal of fMRI) is compared at each specific brain location between different groups [105]. Univariate techniques are generally designed to detect group differences, and have limited utility to perform disease classification at the individual level [106]. Therefore, advanced statistical modeling approaches and networkbased approaches are increasingly applied to "big datasets", such as those generated in neuroimaging, to generate robust discrimination models for biomarker development [107].

\section{Applying Advanced Statistical Approaches}

Machine learning is the science of applying learning algorithms to solve complex problems. In essence, the software is designed to "learn" patterns from the data to classify patients into categories (e.g., diseased or not diseased). The approach consists of 2 stages: 1) the training dataset is used to identify key features and build a statistical model for discrimination; and 2) the validation dataset is used to test the statistical model built from the first stage to assess generalizability to a wider population [108]. Specifically, the statistical model predicts the presence or absence of disease at an individual level.

In comparison to univariate analysis, machine learning methods allow for the incorporation of a large number of potential variables, resulting in multivariate pattern analysis. In such an analysis, data do not have to meet criteria of significance required using univariate models, thereby incorporating statistically insignificant information that is nonetheless important for the classification of disease [107]. As an example, machine learning was compared with univariate techniques for classification of patients with ALS from healthy controls, using resting-state fMRI data (i.e., a phase 2 diagnostic study). The machine learning algorithm had a higher classification accuracy of $72 \%$ compared with univariate analysis, which had a classification accuracy of $54 \%$ [109]. Although this example used only one MRI modality, the goal would be to incorporate several neuroimaging modalities. Furthermore, network-based system analyses can be integrated into machine learning algorithms.

There are inherent limitations to machine learning. For example, the resulting model may be too finely tuned to the training set, producing a nongeneralizable model. Machine learning also has difficulty handling and compensating for noise in data. Nevertheless, machine learning will be a key component in development of diagnostic neuroimaging biomarkers for ALS, as it can incorporate and process a large number of potentially important data, and perform predictions at an individual level. 


\section{Multidimensional Diagnostic Study Examples}

There is much overlap of imaging results between patients with ALS and healthy controls reported in phase 1 studies using various neuroimaging techniques. Thus, it is becoming increasingly clear that development of a neuroimaging biomarker will require the acquisition and analysis of multiple imaging parameters.

There are several examples of multimodal imaging studies in the literature. Filipinni et al. [40] studied 24 patients with longstanding ALS and 24 age- and sex-matched healthy controls. Using DTI and high-resolution structural imaging, they reported a diagnostic accuracy of $92 \%$. Work by our own group reported $93 \%$ diagnostic accuracy using DTI and MRS to classify 29 patients with longstanding ALS and 30 age- and sex-matched healthy controls (Fig. 4) [93]. Van Laere et al. [68] studied 81 patients with a suspected diagnosis of ALS and compared them with 20 healthy controls using $\left[{ }^{18}\right.$ F]FDG PET imaging. Machine learning methods were used to analyze multiple regions of the brain, resulting in a diagnostic accuracy of $95 \%$. In another $\left[{ }^{18}\right.$ F]FDG PET study, Pagani et al. [69] studied 195 patients with ALS and compared them with 40 healthy controls, resulting in a diagnostic accuracy of $95 \%$.

It is important to evaluate the quality of any diagnostic phase study to determine potential biases that may affect the generalizability of results to a larger, external population. Many biomedical journals recommend that the Standards for

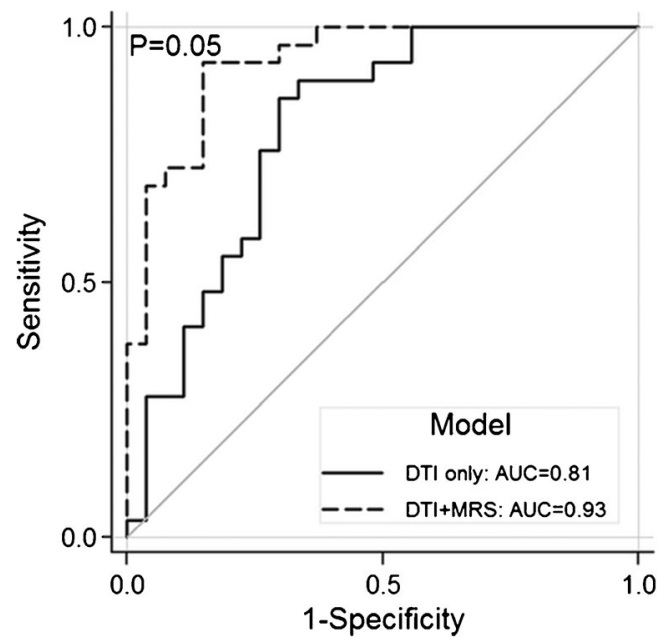

Fig. 4 Receiver operator curve demonstrating significant increases in added utility of combining magnetic resonance spectroscopy (MRS) and diffusion tensor imagin (DTI) for amyotropic lateral sclerosis. Solid line represents the model using only DTI fractional anisotropy (FA) values of the corticospinal tracts. Dashed line represents the model using DTI FA values of the corticospinal tracts combined with MRS data of the motor cortex. AUC = area under the curve. Reproduced with permission from Foerster BR, Carlos RC, Dwamena BA, et al. Multimodal MRI as a diagnostic biomarker for amytrophic lateral sclerosis. Ann Clin Transl Neurol 2014;1:1-8. Copyright (C) 2014 Wiley, Inc
Reporting of Diagnostic accuracy checklist be used to improve the accuracy and completeness of study reporting [110]. As an example, the Standards for Reporting of Diagnostic checklist for the abovementioned 4 studies is presented in Table 1. It is important to note that none of these studies validated the reported test performance in an independent sample, which is a critical step in establishing potential generalizability of the proposed approaches.

\section{Multicenter Efforts}

As illustrated above, there have been several relatively small studies using neuroimaging methods to study ALS. There are increasing efforts to combine imaging datasets across centers, generating "retrospective" multicenter imaging studies. The Neuroimaging Society in ALS is a consortium of international researchers focused on applying imaging techniques to better understand the pathophysiology of the disease and to develop much needed biomarkers for ALS [111]. Efforts of the group include standardization of imaging protocols and the development of an imaging databank to combine datasets. Challenges to be overcome include heterogeneity of both clinical and imaging data [112]. An encouraging example comes from the Alzheimer's disease literature in which machine learning methods were applied to analyze structural MRI data collected from multiple centers. The machine learning model was able to classify the patients with different subtypes of dementia with high accuracy [113]. Prospective multicenter trials will certainly be a significant step forward in the development of neuroimaging biomarkers for ALS.

\section{Future Directions}

Several limitations exist in the literature that must be addressed in future investigations regarding the potential of neuroimaging to serve as a diagnostic biomarker. To perform assessments at an individual level, normative data from a large number of healthy controls are required. Normative data can also be used as a first step to explore heterogeneity of imaging parameters across different imaging sites and equipment. Heterogeneity in collected data needs to determined and addressed statistically to ensure the generalizability of any proposed neuroimaging biomarker for ALS. As discussed above, additional efforts are required to understand the true effect size of neuroimaging metrics in patients with early disease. Longitudinal studies are required for phase 3 studies, in which patients with suspected disease are enrolled and followed clinically to assess for conversion to definite disease status. Last, consideration should be given to incorporating other nonimaging test results, such as serum and cerebrospinal fluid markers. 
Table 1 Standards for Reporting of Diagnostic checklist for diagnostic multidimensional imaging studies

\begin{tabular}{|c|c|c|c|c|}
\hline & $\begin{array}{l}\text { Filippini et al. } 2010 \\
\text { [40] }\end{array}$ & $\begin{array}{l}\text { Foerster et al. } 2014 \\
\text { [93] }\end{array}$ & Van Laere et al. 2014 [68] & $\begin{array}{l}\text { Pagani et al. } 2014 \\
\text { [69] }\end{array}$ \\
\hline Article identified as diagnostic accuracy study & No & No & No & No \\
\hline State study aims & Yes & Yes & Yes & Yes \\
\hline Study population described & Yes & Yes & Yes & Yes \\
\hline Describe participant recruitment & Yes & Yes & Yes & Yes \\
\hline Describe participant sampling & Yes & No & Yes & No \\
\hline Describe data collection & Yes & Yes & Yes & Yes \\
\hline Describe reference standard & Yes & Yes & Yes & Yes \\
\hline Describe technical specifications & Yes & Yes & Yes & Yes \\
\hline $\begin{array}{l}\text { Describe definition of cut-offs and index } \\
\text { test results }\end{array}$ & No & Yes & Yes & Yes \\
\hline $\begin{array}{l}\text { Describe the number and expertise of persons } \\
\text { reading tests }\end{array}$ & Yes & Yes & Yes & No \\
\hline Describe whether blinding was used & No & Yes & No & No \\
\hline $\begin{array}{l}\text { Describe methods for calculating diagnostic } \\
\text { accuracy measures }\end{array}$ & Yes & Yes & Yes & Yes \\
\hline Report dates of study & No & No & Yes & Yes \\
\hline $\begin{array}{l}\text { Report clinical and demographic information } \\
\text { of subjects }\end{array}$ & Yes & Yes & Yes & Yes \\
\hline Report subject drop-outs & Yes & Yes & Yes & Yes \\
\hline $\begin{array}{l}\text { Report time interval between index test and } \\
\text { reference standard }\end{array}$ & Yes & Yes & Yes & Yes \\
\hline Report distribution of disease & Yes & Yes & Yes & Yes \\
\hline Report estimates of diagnostic accuracy & Yes & Yes & Yes & Yes \\
\hline $\begin{array}{l}\text { Report measures of uncertainty in diagnostic } \\
\text { accuracy }\end{array}$ & No & Yes & No & Yes \\
\hline Report how indeterminate results were handled & Yes & Yes & Yes & Yes \\
\hline $\begin{array}{l}\text { Discuss clinical applicability of the study } \\
\text { findings }\end{array}$ & Yes & Yes & Yes & Yes \\
\hline
\end{tabular}

\section{How Can Imaging Contribute to Therapy?}

Most clinical trials use the revised El Escorial criteria to identify patients for inclusion with "Clinically Definite ALS", "Clinically Probable ALS", or "Clinically Probable Laboratory-supported ALS" [114]. However, some patients do not fulfill inclusion criterion owing to a lack of upper motor neuron signs at the clinical examination. In these cases, an imaging marker to objectively assess upper motor neuron involvement could contribute to diagnosis, much like electromyography serves as an objective marker for lower motor neuron involvement [5]. Such an approach could facilitate an earlier diagnosis of ALS, and enable therapeutic interventions at an earlier stage of the disease process.

An extension of this concept is to use imaging to study genetic carriers of the disease who are presymptomatic. Genetic studies have identified the genetic basis of $60-70 \%$ of individuals with familial ALS, resulting in recent years in a substantial increase in the number of identifiable presymptomatic carriers of ALS gene mutations [115, 116]. Studies of presymptomatic carriers hold promise to reveal the underlying pathophysiology changes that occur before clinical symptoms are evident. For example, previous imaging studies including presymptomatic carriers of the SOD1 gene have shown alterations, suggesting ALS pathology may be present years before clinical symptoms occur $[53,117,118]$. However, it is uncertain to what extent ALS caused by SOD1 pathology can be generalized to patients with sporadic ALS. Furthermore, longitudinal studies of presymptomatic carriers are lacking.

In addition to aiding ALS diagnosis, neuroimaging studies may increase our knowledge of fundamental disease processes, which may differ on an individual patient basis [119]. An important recent insight supported by several imaging studies has been the potential spread of disease along connections in brain networks [48, 92]. Furthermore, imaging data are increasingly supported by cellular and molecular studies. Such efforts will help facilitate the development of treatments aimed at inhibiting the spread of disease. Another promising direction of research is the exploration of brain phenotypes of patients within the clinical spectrum of ALS-FTD. This brain phenotype may depend upon the underlying pathology, for example TDP-43, TAU, or SOD1. Furthermore, increasing 
insights into various pathophysiologic processes occurring in ALS may enable targeted therapies. Imaging could be used to evaluate therapeutic responses along these different pathophysiologic cascades. Imaging markers for disease progression in ALS would facilitate drug discovery in clinical trials by detecting treatment responses that are not detected by clinical measures or by identifying a differential response in a subgroup of patients $[8,120,121]$.

\section{Conclusions}

There is growing interest in using neuroimaging to improve our understanding of ALS. Neuroimaging holds promise to facilitate diagnosis by providing objective upper motor neuron markers, as well as markers for extramotor brain involvement. In addition to diagnostic value, imaging metrics may be used to monitor disease progression, and aid clinical trials and drug discovery.

Neuroimaging has provided compelling evidence that ALS is a complex disease affecting different regions of the brain and resulting in changes of structure, receptors, metabolism, and connectivity. We have highlighted the current leading neuroimaging modalities that could potentially provide diagnostic biomarkers. Imaging can interrogate pathophysiology noninvasively and at early stages, particularly in patients who do not have convincing clinical examination findings, providing an opportunity for early therapeutic intervention and better patient outcomes.

Given the complexity of the disease, a multimodal approach and advanced statistical/network-based analyses will be required to develop meaningful diagnostic biomarkers. A step-wise approach to the development of diagnostic biomarkers for ALS is important given the challenges of studying such a heterogeneous, relatively fast-progressing, and fatal disease. Last, multicenter trials will be needed to establish generalizability of any proposed biomarker to clinical practice.

Acknowledgments Dr. Bradley Foerster has received support from NIH Grant R01 NS082301-01.

Required Author Forms Disclosure forms provided by the authors are available with the online version of this article.

\section{References}

1. Kiernan MC, Vucic S, Cheah BC, et al. Amyotrophic lateral sclerosis. Lancet 2011;377:942-955.

2. Ravits JM, La Spada AR. ALS motor phenotype heterogeneity, focality, and spread: deconstructing motor neuron degeneration. Neurology 2009;73:805-811.
3. Chio A, Calvo A, Moglia C, Mazzini L, Mora G. Phenotypic heterogeneity of amyotrophic lateral sclerosis: a population based study. J Neurol Neurosurg Psychiatry 2011;82:740-746.

4. Al-Chalabi A, Hardiman O. The epidemiology of ALS: a conspiracy of genes, environment and time. Nat Rev Neurol 2013;9:617628.

5. Eisen A, Swash M. Clinical neurophysiology of ALS. Clin Neurophysiol 2001;112:2190-2201.

6. Tartaglia MC, Rowe A, Findlater K, Orange JB, Grace G, Strong MJ. Differentiation between primary lateral sclerosis and amyotrophic lateral sclerosis: examination of symptoms and signs at disease onset and during follow-up. Arch Neurol 2007;64:232-236.

7. Riku Y, Atsuta N, Yoshida M, et al. Differential motor neuron involvement in progressive muscular atrophy: a comparative study with amyotrophic lateral sclerosis. BMJ Open 2014;4:e05213.

8. Turner MR, Kiernan MC, Leigh PN, Talbot K. Biomarkers in amyotrophic lateral sclerosis. Lancet Neurol 2009;8:94-109.

9. Paganoni S, Macklin EA, Lee A, et al. Diagnostic timelines and delays in diagnosing amyotrophic lateral sclerosis (ALS). Amyotroph Lateral Scler Frontotemporal Degener 2014;15:453456.

10. Ringholz GM, Appel SH, Bradshaw M, Cooke NA, Mosnik DM, Schulz PE. Prevalence and patterns of cognitive impairment in sporadic ALS. Neurology 2005;65:586-590.

11. Filippi M, Rocca MA. MR imaging of multiple sclerosis. Radiology 2011;259:659-681.

12. Ashburner J, Friston KJ. Voxel-based morphometry - the methods. Neuroimage 2000;11:805-821.

13. Fischl B, Dale AM. Measuring the thickness of the human cerebral cortex from magnetic resonance images. Proc Natl Acad Sci U S A 2000;97:11050-11055.

14. Chenevert TL, Brunberg JA, Pipe JG. Anisotropic diffusion in human white matter: demonstration with MR techniques in vivo. Radiology 1990;177:401-405.

15. Chang JL, Lomen-Hoerth C, Murphy J, et al. A voxel-based morphometry study of patterns of brain atrophy in ALS and ALS/ FTLD. Neurology 2005;65:75-80.

16. Grosskreutz J, Kaufmann J, Fradrich J, Dengler R, Heinze HJ, Peschel T. Widespread sensorimotor and frontal cortical atrophy in amyotrophic lateral sclerosis. BMC Neurol 2006;6:6-17.

17. Kassubek J, Unrath A, Huppertz HJ, et al. Global brain atrophy and corticospinal tract alterations in ALS, as investigated by voxelbased morphometry of 3-D MRI. Amyotroph Lateral Scler Other Motor Neuron Disord 2005;6:213-220.

18. Turner MR, Hammers A, Allsop J, et al. Volumetric cortical loss in sporadic and familial amyotrophic lateral sclerosis. Amyotroph Lateral Scler 2007;8:343-347.

19. Agosta F, Pagani E, Rocca MA, et al. Voxel-based morphometry study of brain volumetry and diffusivity in amyotrophic lateral sclerosis patients with mild disability. Hum Brain Mapp 2007;28:14301438.

20. Bede P, Bokde A, Elamin M, et al. Grey matter correlates of clinical variables in amyotrophic lateral sclerosis (ALS): a neuroimaging study of ALS motor phenotype heterogeneity and cortical focality. J Neurol Neurosurg Psychiatry 2013;84:766-773.

21. Mezzapesa DM, Ceccarelli A, Dicuonzo F, et al. Whole-brain and regional brain atrophy in amyotrophic lateral sclerosis. AJNR Am J Neuroradiol 2007;28:255-259.

22. Tsujimoto M, Senda J, Ishihara $T$, et al. Behavioral changes in early ALS correlate with voxel-based morphometry and diffusion tensor imaging. J Neurol Sci 2011;307:34-40.

23. Abrahams S, Goldstein LH, Suckling J, et al. Frontotemporal white matter changes in amyotrophic lateral sclerosis. J Neurol 2005;252: 321-331.

24. Rajagopalan V, Yue GH, Pioro EP. Do preprocessing algorithms and statistical models influence voxel-based morphometry (VBM) 
results in amyotrophic lateral sclerosis patients? A systematic comparison of popular VBM analytical methods. J Magn Reson Imaging 2014;40:662-667.

25. Chen Z, Ma L. Grey matter volume changes over the whole brain in amyotrophic lateral sclerosis: a voxel-wise meta-analysis of voxel based morphometry studies. Amyotroph Lateral Scler 2010;11:549554.

26. Rajagopalan V, Pioro EP. Distinct patterns of cortical atrophy in ALS patients with or without dementia: an MRI VBM study. Amyotroph Lateral Scler Frontotemporal Degener 2014;15:216225.

27. Menke RA, Korner S, Filippini N, et al. Widespread grey matter pathology dominates the longitudinal cerebral MRI and clinical landscape of amyotrophic lateral sclerosis. Brain 2014;137:25462555.

28. Agosta F, Valsasina P, Riva N, et al. The cortical signature of amyotrophic lateral sclerosis. PloS One 2012;7:e42816.

29. Schuster C, Kasper E, Machts J, et al. Focal thinning of the motor cortex mirrors clinical features of amyotrophic lateral sclerosis and their phenotypes: a neuroimaging study. J Neurol 2013;260:28562864.

30. Verstraete E, Veldink JH, Hendrikse J, Schelhaas HJ, van den Heuvel MP, van den Berg LH. Structural MRI reveals cortical thinning in amyotrophic lateral sclerosis. J Neurol Neurosurg Psychiatry 2012;83:383-388.

31. Roccatagliata L, Bonzano L, Mancardi G, Canepa C, Caponnetto C. Detection of motor cortex thinning and corticospinal tract involvement by quantitative MRI in amyotrophic lateral sclerosis. Amyotroph Lateral Scler 2009;10:47-52.

32. Walhout R, Westeneng HJ, Verstraete E, et al. Cortical thickness in ALS: towards a marker for upper motor neuron involvement. J Neurol Neurosurg Psychiatry 2015;86:288-294.

33. Rajagopalan V, Liu Z, Allexandre D, et al. Brain white matter shape changes in amyotrophic lateral sclerosis (ALS): a fractal dimension study. PloS One 2013;8:e73614.

34. Smith MC. Nerve fibre degeneration in the brain in amyotrophic lateral sclerosis. J Neurol Neurosurg Psychiatry 1960;23:269-282.

35. Song SK, Sun SW, Ju WK, Lin SJ, Cross AH, Neufield AH. Diffusion tensor imaging detects and differentiates axon and myelin degeneration in mouse optic nerve after retinal ischemia. Neuroimage 2003;20:1714-1722.

36. Foerster BR, Welsh RC, Feldman EL. 25 years of neuroimaging in amyotrophic lateral sclerosis. Nat Rev Neurol 2013;9:513-524.

37. van der Graaff MM, Sage CA, Caan MW, et al. Upper and extramotoneuron involvement in early motoneuron disease: a diffusion tensor imaging study. Brain 2011;134:1211-1228.

38. Agosta F, Pagani E, Petrolini M, et al. Assessment of white matter tract damage in patients with amyotrophic lateral sclerosis: a diffusion tensor MR imaging tractography study. AJNR Am J Neuroradiol 2010;31:1457-1461.

39. Metwalli NS, Benatar M, Nair G, Usher S, Hu X, Carew JD. Utility of axial and radial diffusivity from diffusion tensor MRI as markers of neurodegeneration in amyotrophic lateral sclerosis. Brain Res 2010;1348:156-164.

40. Filippini N, Douaud G, Mackay CE, Knight S, Talbot K, Turner MR. Corpus callosum involvement is a consistent feature of amyotrophic lateral sclerosis. Neurology 2010;75:1645-1652.

41. Chapman MC, Jelsone-Swain L, Johnson TD, Gruis KL, Welsh RC. Diffusion tensor MRI of the corpus callosum in amyotrophic lateral sclerosis. J Magn Reson Imaging 2014;39:641-647.

42. Rajagopalan V, Yue GH, Pioro EP. Brain white matter diffusion tensor metrics from clinical 1.5T MRI distinguish between ALS phenotypes. J Neurol 2013;260:2532-2540.

43. Thivard L, Pradat PF, Lehericy S, et al. Diffusion tensor imaging and voxel based morphometry study in amyotrophic lateral sclerosis: relationships with motor disability. J Neurol Neurosurg Psychiatry 2007;78:889-892.

44. Abe O, Yamada H, Masutani Y, et al. Amyotrophic lateral sclerosis: diffusion tensor tractography and voxel-based analysis. NMR Biomed 2004;17:411-416.

45. Sach M, Winkler G, Glauche V, et al. Diffusion tensor MRI of early upper motor neuron involvement in amyotrophic lateral sclerosis. Brain 2004;127:340-350.

46. Prell T, Peschel T, Hartung V, et al. Diffusion tensor imaging patterns differ in bulbar and limb onset amyotrophic lateral sclerosis. Clin Neurol Neurosurg 2013;115:1281-1287.

47. Sharma KR, Sheriff S, Maudsley A, Govind V. Diffusion tensor imaging of basal ganglia and thalamus in amyotrophic lateral sclerosis. J Neuroimaging 2013;23:368-374.

48. Kassubek J, Muller HP, Del Tredici K, et al. Diffusion tensor imaging analysis of sequential spreading of disease in amyotrophic lateral sclerosis confirms patterns of TDP-43 pathology. Brain 2014; 137:1733-1740.

49. Pike VW. PET radiotracers: crossing the blood-brain barrier and surviving metabolism. Trends Pharmacol Sci 2009;30:431-440.

50. Cistaro A, Cuccurullo V, Quartuccio N, Pagani M, Valentini MC, Mansi L. Role of PET and SPECT in the study of amyotrophic lateral sclerosis. Biomed Res Int 2014;2014:237437.

51. Castillo M, Kwock L, Mukherji SK. Clinical applications of proton MR spectroscopy. AJNR Am J Neuroradiol 1996;17:1-15.

52. Mullins PG, McGonigle DJ, O'Gorman RL, et al. Current practice in the use of MEGA-PRESS spectroscopy for the detection of GABA. NeuroImage 2014;86:43-52.

53. Turner MR, Hammers A, Al-Chalabi A, et al. Distinct cerebral lesions in sporadic and 'D90A' SOD1 ALS: studies with [11C]flumazenil PET. Brain 2005;128:1323-1329.

54. Turner MR, Rabiner EA, Al-Chalabi A, et al. Cortical 5-HT1A receptor binding in patients with homozygous D90A SOD1 vs sporadic ALS. Neurology 2007;68:1233-1235.

55. Turner MR, Cagnin A, Turkheimer FE, et al. Evidence of widespread cerebral microglial activation in amyotrophic lateral sclerosis: an $[11 \mathrm{C}](\mathrm{R})-\mathrm{PK} 11195$ positron emission tomography study. Neurobiol Dis 2004;15:601-609.

56. Turner MR. MRI as a frontrunner in the search for amyotrophic lateral sclerosis biomarkers? Biomark Med 2011;5:79-81.

57. Bowen BC, Pattany PM, Bradley WG, et al. MR imaging and localized proton spectroscopy of the precentral gyrus in amyotrophic lateral sclerosis. AJNR Am J Neuroradiol 2000;21:647-658.

58. Lombardo F, Frijia F, Bongioanni P, et al. Diffusion tensor MRI and MR spectroscopy in long lasting upper motor neuron involvement in amyotrophic lateral sclerosis. Arch Ital Biol 2009;147:69-82.

59. Kalra S, Hanstock CC, Martin WR, Allen PS, Johnston WS. Detection of cerebral degeneration in amyotrophic lateral sclerosis using high-field magnetic resonance spectroscopy. Arch Neurol 2006:63:1144-1148.

60. Bradley WG, Bowen BC, Pattany PM, Rotta F. 1H-magnetic resonance spectroscopy in amyotrophic lateral sclerosis. J Neurol Sci 1999;169:84-86.

61. Han J, Ma L. Study of the features of proton MR spectroscopy ((1)H-MRS) on amyotrophic lateral sclerosis. J Magn Reson Imaging 2010;31:305-308.

62. Foerster BR, Pomper MG, Callaghan BC, et al. An imbalance between excitatory and inhibitory neurotransmitters in amyotrophic lateral sclerosis revealed by use of 3-T proton magnetic resonance spectroscopy. JAMA Neurol 2013;70:1009-1016.

63. Foerster BR, Callaghan BC, Petrou M, Edden RA, Chenevert TL, Feldman EL. Decreased motor cortex gamma-aminobutyric acid in amyotrophic lateral sclerosis. Neurology 2012;78:1596-1600.

64. Kiernan MC, Petri S. Hyperexcitability and amyotrophic lateral sclerosis. Neurology 2012;78:1544-1545. 
65. Gore JC. Principles and practice of functional MRI of the human brain. J Clin Invest 2003;112:4-9.

66. Kew JJ, Leigh PN, Playford ED, et al. Cortical function in amyotrophic lateral sclerosis. A positron emission tomography study. Brain 1993;116:655-680.

67. Cistaro A, Valentini MC, Chio A, et al. Brain hypermetabolism in amyotrophic lateral sclerosis: a FDG PET study in ALS of spinal and bulbar onset. Eur J Nuc Med Mol Imaging 2012;39:251-259.

68. Van Laere K, Vanhee A, Verschueren J, et al. Value of 18 fluorodeoxyglucose-positron-emission tomography in amyotrophic lateral sclerosis: a prospective study. JAMA Neurol 2014;71: 553-561.

69. Pagani M, Chio A, Valentini MC, et al. Functional pattern of brain FDG-PET in amyotrophic lateral sclerosis. Neurology 2014;83: 1067-1074.

70. Schoenfeld MA, Tempelmann C, Gaul C, et al. Functional motor compensation in amyotrophic lateral sclerosis. J Neurol 2005;252: 944-952.

71. Mohammadi B, Kollewe K, Samii A, Dengler R, Munte TF. Functional neuroimaging at different disease stages reveals distinct phases of neuroplastic changes in amyotrophic lateral sclerosis. Hum Brain Mapp 2011;32:750-758.

72. Raaphorst J, van Tol MJ, Groot PF, et al. Prefrontal involvement related to cognitive impairment in progressive muscular atrophy. Neurology 2014;83:818-825.

73. Sporns O, Zwi JD. The small world of the cerebral cortex. Neuroinformatics 2004;2:145-162.

74. Lowe MJ, Dzemidzic M, Lurito JT, Mathews VP, Phillips MD. Correlations in low-frequency BOLD fluctuations reflect corticocortical connections. Neuroimage 2000;12:582-587.

75. Nir Y, Mukamel R, Dinstein I, et al. Interhemispheric correlations of slow spontaneous neuronal fluctuations revealed in human sensory cortex. Nat Neurosci 2008;11:1100-1108.

76. Shmuel A, Leopold DA. Neuronal correlates of spontaneous fluctuations in fMRI signals in monkey visual cortex: implications for functional connectivity at rest. Hum Brain Mapp 2008;29:751-761.

77. Mohammadi B, Kollewe K, Samii A, Krampfl K, Dengler R, Munte TF. Changes of resting state brain networks in amyotrophic lateral sclerosis. Exp Neurol 2009;217:147-153.

78. Greicius MD, Krasnow B, Reiss AL, Menon V. Functional connectivity in the resting brain: a network analysis of the default mode hypothesis. Proc Natl Acad Sci U S A 2003;100:253-258.

79. van den Heuvel MP, Mandl RC, Kahn RS, Hulshoff Pol HE. Functionally linked resting-state networks reflect the underlying structural connectivity architecture of the human brain. Hum Brain Mapp 2009;30:3127-3141.

80. De Luca M, Smith S, De Stefano N, Federico A, Matthews PM. Blood oxygenation level dependent contrast resting state networks are relevant to functional activity in the neocortical sensorimotor system. Exp Brain Res 2005;167:587-594.

81. Jelsone-Swain LM, Fling B, Seidler RD, Hovatter R, Gruis K, Welsh RC. Reduced interhemispheric functional connectivity in the motor cortex during rest in limb-onset amyotrophic lateral sclerosis. Front Syst Neurosci 2010;4:158.

82. Tedeschi G, Trojsi F, Tessitore A, et al. Interaction between aging and neurodegeneration in amyotrophic lateral sclerosis. Neurobiol Aging 2012;33:886-898

83. Agosta F, Valsasina P, Absinta M, et al. Sensorimotor functional connectivity changes in amyotrophic lateral sclerosis. Cereb Cortex 2011;21:2291-2298.

84. Douaud G, Filippini N, Knight S, Talbot K, Turner MR. Integration of structural and functional magnetic resonance imaging in amyotrophic lateral sclerosis. Brain 2011;134:3470-3479.

85. Zhou F, Xu R, Dowd E, Zang Y, Gong H, Wang Z. Alterations in regional functional coherence within the sensory-motor network in amyotrophic lateral sclerosis. Neurosci Lett 2014;558:192-196.
86. Schmidt R, Verstraete E, de Reus MA, Veldink JH, van den Berg LH, van den Heuvel MP. Correlation between structural and functional connectivity impairment in amyotrophic lateral sclerosis. Hum Brain Mapp 2014;35:4386-4395.

87. Agosta F, Canu E, Valsasina P, et al. Divergent brain network connectivity in amyotrophic lateral sclerosis. Neurobiol Aging 2013;34:419-427.

88. Trojsi F, Esposito F, de Stefano M, et al. Functional overlap and divergence between ALS and bvFTD. Neurobiol Aging 2015;36: 413-423.

89. Proudfoot M, Woolrich MW, Nobre AC, Turner MR. Magnetoencephalography. Pract Neurol 2014;14:336-343.

90. Verstraete E, Veldink JH, Mandl RC, van den Berg LH, van den Heuvel MP. Impaired structural motor connectome in amyotrophic lateral sclerosis. PLoS One 2011;6:e24239.

91. Buchanan CR, Pettit LD, Storkey AJ, Abrahams S, Bastin ME. Reduced structural connectivity within a prefrontal-motorsubcortical network in amyotrophic lateral sclerosis. J Magn Reson Imaging 2014;36;1075-1082.

92. Verstraete E, Veldink JH, van den Berg LH, van den Heuvel MP. Structural brain network imaging shows expanding disconnection of the motor system in amyotrophic lateral sclerosis. Hum Brain Mapp 2014;35:1351-1361.

93. Foerster BR, Carlos RC, Dwamena BA, et al. Multimodal MRI as a diagnostic biomarker for amyotrophic lateral sclerosis. Ann Clin Transl Neurol 2014;1:107-114.

94. Gluud C, Gluud LL. Evidence based diagnostics. BMJ 2005;330: 724-726.

95. Sackett DL, Haynes RB. The architecture of diagnostic research. BMJ 2002;324:539-541.

96. "Value in Knowing" Poll. Harris Interactive Inc., 2013.

97. Cosottini M, Giannelli M, Vannozzi F, et al. Evaluation of corticospinal tract impairment in the brain of patients with amyotrophic lateral sclerosis by using diffusion tensor imaging acquisition schemes with different numbers of diffusion-weighting directions. J Comput Assist Tomogr 2010;34:746-750.

98. Blain CR, Brunton S, Williams VC, et al. Differential corticospinal tract degeneration in homozygous 'D90A' SOD-1 ALS and sporadic ALS. J Neurol Neurosurg Psychiatry 2011;82:843-849.

99. Graham JM, Papadakis N, Evans J, et al. Diffusion tensor imaging for the assessment of upper motor neuron integrity in ALS. Neurology 2004;63:2111-2119.

100. Nelles M, Block W, Traber F, Wullner U, Schild HH, Urbach H. Combined $3 \mathrm{~T}$ diffusion tensor tractography and ${ }^{1} \mathrm{H}-\mathrm{MR}$ spectroscopy in motor neuron disease. AJNR Am J Neuroradiol 2008;29: 1708-1714.

101. Pyra T, Hui B, Hanstock C, et al. Combined structural and neurochemical evaluation of the corticospinal tract in amyotrophic lateral sclerosis. Amyotroph Lateral Scler 2010;11:157-165.

102. Foerster BR, Dwamena BA, Petrou M, Carlos RC, Callaghan BC, Pomper MG. Diagnostic accuracy using diffusion tensor imaging in the diagnosis of ALS: a meta-analysis. Acad Radiol 2012;19:10751086.

103. Foerster BR, Dwamena BA, Petrou M, et al. Diagnostic accuracy of diffusion tensor imaging in amyotrophic lateral sclerosis: a systematic review and individual patient data meta-analysis. Acad Radiol 2013;20:1099-1106.

104. Rothstein JD. Current hypotheses for the underlying biology of amyotrophic lateral sclerosis. Ann Neurol 2009;65:S3-S9.

105. Friston KJ. Imaging cognitive anatomy. Trends Cogn Sci 1997;1: 21-27.

106. Vemuri P, Gunter JL, Senjem ML, et al. Alzheimer's disease diagnosis in individual subjects using structural MR images: validation studies. Neuroimage 2008;39:1186-1197.

107. Orru G, Pettersson-Yeo W, Marquand AF, Sartori G, Mechelli A. Using support vector machine to identify imaging biomarkers of 
neurological and psychiatric disease: a critical review. Neurosci Biobehav Rev 2012;36:1140-1152.

108. Wang SJ, Summers RM. Machine learning and radiology. Med Image Anal 2012;16:933-951.

109. Welsh RC, Jelsone-Swain LM, Foerster BR. The utility of independent component analysis and machine learning in the identification of the amyotrophic lateral sclerosis diseased brain. Front Hum Neurosci 2013;7:251.

110. Bossuyt PM, Reitsma JB, Bruns DE, et al. The STARD statement for reporting studies of diagnostic accuracy: explanation and elaboration. Ann Intern Med 2003;138:W1-12.

111. Turner MR, Grosskreutz J, Kassubek J, et al. Towards a neuroimaging biomarker for amyotrophic lateral sclerosis. Lancet Neurol 2011;10:400-403.

112. Pradat PF and El Mendili MM. Neuroimaging to investigate multisystem involvement and provide biomarkers in amyotrophic lateral sclerosis. Biomed Res Int 2014;2014:467560.

113. Kloppel S, Stonnington CM, Chu C, et al. Automatic classification of MR scans in Alzheimer's disease. Brain 2008;131:681-689.

114. Brooks BR, Miller RG, Swash M, Munsat TL. El Escorial revisited: revised criteria for the diagnosis of amyotrophic lateral sclerosis.
Amyotroph Lateral Scler Other Motor Neuron Disord 2000;1:293299.

115. Renton AE, Chio A, Traynor BJ. State of play in amyotrophic lateral sclerosis genetics. Nat Neurosci 2014;17:17-23.

116. Su XW, Broach JR, Connor JR, Gerhard GS, Simmons Z. Genetic heterogeneity of amyotrophic lateral sclerosis: implications for clinical practice and research. Muscle Nerve 2014;49:786-803.

117. $\mathrm{Ng} \mathrm{MC,} \mathrm{Ho} \mathrm{JT,} \mathrm{Ho} \mathrm{SL,} \mathrm{et} \mathrm{al.} \mathrm{Abnormal} \mathrm{diffusion} \mathrm{tensor} \mathrm{in}$ nonsymptomatic familial amyotrophic lateral sclerosis with a causative superoxide dismutase 1 mutation. J Magn Reson Imaging 2008;27:8-13.

118. Carew JD, Nair G, Andersen PM, et al. Presymptomatic spinal cord neurometabolic findings in SOD1-positive people at risk for familial ALS. Neurology 2011;77:1370-1375.

119. Bede P, Hardiman O. Lessons of ALS imaging: pitfalls and future directions - a critical review. Neuroimage Clin 2014;4:436-443.

120. Turner MR, Hardiman O, Benatar M, et al. Controversies and priorities in amyotrophic lateral sclerosis. Lancet Neurol 2013;12:310-322.

121. Winhammar JM, Rowe DB, Henderson RD, Kiernan MC. Assessment of disease progression in motor neuron disease. Lancet Neurol 2005;4:229-238. 